\title{
MEAN VALUES AND NON-PERIODIC PRESSURE IN CONVECTION PROBLEMS BETWEEN PLATES OR WITH STRESS-FREE BOUNDARIES
}

\author{
Burkhard J. SchmitT ANd Wolf VON WAHL
}

When considering the Oberbeck-Boussinesq equations in an infinite layer it is mostly assumed that the pressure $\pi$ is periodic in the plane, whereas the equations only require $\nabla \pi$ to be periodic. We study here the influence the general admissible form of the pressure may have on the velocity field $u$ below the onset of convection, a question which is closely connected with the mean flow. This is a vector field which depends on $z$ only and which is given by the mean values of $u_{x}, u_{y}, u_{z}$ over the plane periodicity cell. - The mean value of $u$ over the layer is constant under stress-free boundary conditions and periodic pressure. If this constant $c$ is not 0 there is in most cases no longer an exchange of stability on the onset for the linearization around c. We study its spectrum on the onset.

\section{Introduction, Notations.}

We consider an incompressible viscous fluid in an infinite layer $\mathbb{R}^{2} \times\left(-\frac{1}{2}, \frac{1}{2}\right)$ heated from below. The perturbations of the motionless state are governed by the Oberbeck-Boussinesq equations

$$
\left\{\begin{array}{r}
\partial_{t} \mathbf{u}-\Delta \mathbf{u}+\mathbf{u} \cdot \nabla \mathbf{u}-\sqrt{R} \vartheta \mathbf{k}+\nabla \pi=0, \\
\nabla \cdot \mathbf{u}=0, \\
\operatorname{Pr} \partial_{t} \vartheta-\Delta \vartheta+\operatorname{Pr} \mathbf{u} \cdot \nabla \vartheta-\sqrt{R} u_{z}=0 .
\end{array}\right.
$$

$R$ is the Rayleigh-number and is proportional to the intensity of heating, $\operatorname{Pr}$ is the Prandtl-number. $R$ serves as control parameter. $\mathbf{u}=\left(u_{x}, u_{y}, u_{z}\right)^{\mathrm{T}}=$ $\left(u_{1}, u_{2}, u_{3}\right)^{\mathrm{T}}$ is the velocity field of the perturbation, $\pi$ its pressure and $\vartheta$ is the fluctuation of the temperature from a static state. We consider (I.1) under stress-free boundaries

$$
\partial_{z} u_{x}=\partial_{z} u_{y}=u_{z}=\vartheta=0 \quad \text { at } z= \pm \frac{1}{2}
$$

as well as under rigid ones

$$
\mathbf{u}=0, \vartheta=0 \quad \text { at } z= \pm \frac{1}{2} .
$$


Here $\mathbf{x}=(x, y, z)^{\mathrm{T}} \in \mathbb{R}^{2} \times\left[-\frac{1}{2}, \frac{1}{2}\right]$. The solutions $(\mathbf{u}, \pi, \vartheta)$ of (I.1) shall have the property that at least $\mathbf{u}, \vartheta$ are periodic with respect to the plane variables $x, y$. Let us assume that $\mathcal{P}=\left(-\frac{\pi}{\alpha}, \frac{\pi}{\alpha}\right) \times\left(-\frac{\pi}{\beta}, \frac{\pi}{\beta}\right)$ is the plane periodicity cell with wave numbers $\alpha, \beta$ in $x, y$-direction respectively. $\mathbf{u}, \pi$, $\vartheta$ are assumed to be real valued throughout this paper, although they will be considered in complex function spaces.

As for the pressure only $\nabla \pi$ needs to be periodic. This is what (I.1) requires. In the literature it is in general assumed that the pressure $\pi$ itself is periodic with respect to $\mathcal{P}$. There are however several exceptions which we will touch later. The reason for assuming $\pi$ to be periodic is that when considering perturbations of the motionless state as given by (I.1), the usual stability considerations require $(\nabla \pi, \mathbf{u})_{L^{2}}=0$. Beside by $u_{z}=0$ at $z= \pm 1 / 2$ the latter is ensured by plane periodicity of $\pi$. To eliminate the pressure from the Navier-Stokes part in (I.1) we therefore have applied the projection onto the orthogonal complement to the fields $\nabla \pi$ with $\pi$ periodic. As already mentioned only $\nabla \pi$ has to be periodic. The general form of $\pi$ will be discussed in what follows shortly after these considerations (cf. (I.5) to follow). We now can try to find $\mathbf{u}$ in the orthogonal complement to the fields $\nabla \pi$ with $\nabla \pi$ periodic. As it follows from (I.5) below then

$$
\int_{\mathcal{P} \times\left(-\frac{1}{2}, \frac{1}{2}\right)} u_{x} d x d y d z=\int_{\mathcal{P} \times\left(-\frac{1}{2}, \frac{1}{2}\right)} u_{y} d x d y d z=0
$$

for the perturbation which is not suitable for rigid boundaries in our situation but holds for stress-free boundaries if $\pi$ is periodic and the initial value of $\mathbf{u}$ satisfies this requirement, see (I.3) to follow. Because of these difficulties we will study (I.1) not by means of projections onto the orthogonal complement to gradients but by a different method.

The present paper intends to study the changes in the long time behaviour of the solutions of (I.1) if $\pi$ is no longer periodic. These changes are closely connected with certain mean values of $\mathbf{u}$. Therefore we also pay some attention to the various mean values of $\mathbf{u}$, both under periodic and non-periodic pressure. The essential tool of our analysis is a particular decomposition of any solenoidal field which allows us to separate that parts of the solution of (I.1) which are important at the respective occasions. The results are described in what follows and in a conclusion section at the end of the paper.

In the stress-free case it is common usage to assume that the mean value

$$
\int_{\Omega} \mathbf{u} d x d y d z=0
$$

over the layer $\Omega=\left(-\frac{\pi}{\alpha}, \frac{\pi}{\alpha}\right) \times\left(-\frac{\pi}{\beta}, \frac{\pi}{\beta}\right) \times\left(-\frac{1}{2}, \frac{1}{2}\right)$ and, as already mentioned, 
that $\pi$ is periodic (cf. [GP, WI, SvWI, SvWII]). First of all let us maintain the assumption that $\pi$ is periodic but drop (I.2). Then it follows by integrating (I.1) over $\Omega$ that the mean value of $\mathbf{u}$ over the layer is constant with respect to time, and

$$
\left\{\begin{array}{l}
\frac{1}{|\mathcal{P}|} \int_{\Omega} u_{x}(t, x, y, z) d x d y d z=\frac{1}{|\mathcal{P}|} \int_{\Omega} u_{x}(0, x, y, z) d x d y d z=c_{1} \\
\frac{1}{|\mathcal{P}|} \int_{\Omega} u_{y}(t, x, y, z) d x d y d z=\frac{1}{|\mathcal{P}|} \int_{\Omega} u_{y}(0, x, y, z) d x d y d z=c_{2}
\end{array}\right.
$$

can be prescribed arbitrarily whereas always

$$
\frac{1}{|\mathcal{P}|} \int_{\Omega} u_{z}(t, x, y, z) d x d y d z=\frac{1}{|\mathcal{P}|} \int_{\Omega} u_{z}(0, x, y, z) d x d y d z=0
$$

There is also no problem to construct the unique strong solution of (I.1) with non vanishing mean values $c_{1}, c_{2}$. Again we refer to the poloidal-toroidalmean flow decomposition and [SvWI, WI, WII]. Reduction of the case $\left(c_{1}, c_{2}\right) \neq(0,0)$ to the case (I.2) by replacing $\mathbf{u}$ by $\mathbf{w}=\mathbf{u}-\mathbf{c}$ shows that $(\mathbf{w}, \pi, \vartheta)$ are a perturbation of the constant flow $\mathbf{c}=\left(c_{1}, c_{2}, 0\right)^{\mathrm{T}}, \pi=$ const., $\vartheta=0$ under stress-free boundaries. Using Galileian invariance of (I.1) simultaneously it turns out that $\mathbf{c}$ is unconditionally monotonically energy stable up to criticality. The latter one is represented by the onset of convection as given in [SvWII], and the corresponding critical value of $R$ will subsequently be denoted by $R_{C}=R_{C}(\alpha, \beta)$. The stability properties of $\mathbf{c}$ can of course be recovered by considering in the usual way the disturbances $\mathbf{w}$ (with $\int_{\Omega} \mathbf{w} d x d y d z=0$ ) of $\mathbf{c}$. Theorem 1.1 in [WIII], which gives a necessary and sufficient condition for

$$
\text { energetic Rayleigh-number = critical Rayleigh-number, }
$$

however shows the remarkable effect that in general all eigenvalues of the linearization on the onset become purely imaginary whereas the eigenvalue 0 only occurs if

$$
\frac{c_{1} \alpha}{c_{2} \beta} \quad \text { or } \quad \frac{c_{2} \beta}{c_{1} \alpha} \text { is rational }
$$

and the critical disturbance $\mathbf{w}$ therefore is two-dimensional, cf. [WIII, Sec. 3]. In a sense, the general case of purely imaginary eigenvalues occurring on the onset complements the situation treated in [GS], as now an exchange of stability does not hold. In the case of rigid boundary conditions there is no freedom left to dispose of mean values. 
This relatively simple situation changes drastically when the pressure is no longer $x, y$-periodic. The system (I.1) only requires, as was already mentioned, periodicity of $\nabla \pi$. The general form of the pressure was derived in $[\mathbf{S v W I}]$. It looks like

$$
\pi(t, x, y, z)=\tilde{\pi}(t, x, y, z)+g_{1}(t) x+g_{2}(t) y+d(t, z)
$$

where $\tilde{\pi}$ is periodic in the plane with respect to $\mathcal{P}$ and fulfills $\int_{\mathcal{P}} \tilde{\pi} d x d y=0$. $d$ is subject to the condition

$$
\partial_{z}\left(d+\frac{1}{|\mathcal{P}|} \int_{\mathcal{P}} u_{z}^{2} d x d y\right)=\sqrt{R} \frac{1}{|\mathcal{P}|} \int_{\mathcal{P}} \vartheta d x d y
$$

The two-vector

$$
\mathbf{g}(t)=\left(\begin{array}{l}
g_{1}(t) \\
g_{2}(t)
\end{array}\right)
$$

is arbitrary and depends on $t$ only. Thus $\tilde{\pi}+d$ is that part of $\pi$ which is periodic in the plane whereas $g_{1}(t) x+g_{2}(t) y$ is the non-periodic one. Using the poloidal-toroidal-mean flow decomposition of solenoidal fields there is again no difficulty to solve (I.1) including all terms in (I.5) instead of the periodic one only as we did in [WI, WII]. The proofs in [WI, WII] carry over word by word. Thus from a purely mathematical point of view there is no reason why the periodic case is preferred to the non-periodic one. From a physical point of view the situation looks different. We deal with rigid boundaries first. Taking into consideration the non-periodic part of $\pi$ too we show that the asymptotic behaviour of $\mathbf{u}$ in (I.1) is governed by the mean flow, provided $R<R_{C}$ and provided the deviations from periodicity in $\pi$ are not too large. To be more precise, as $t \rightarrow+\infty$ the velocity field $\mathbf{u}$ behaves like the vector field $\left(f_{1}(t, z), f_{2}(t, z), 0\right)^{\mathrm{T}}$ where

$$
\begin{aligned}
& \partial_{t} f_{1}+\left(-\partial_{z}^{2}\right) f_{1}=-g_{1}(t), \\
& \partial_{t} f_{2}+\left(-\partial_{z}^{2}\right) f_{2}=-g_{2}(t)
\end{aligned}
$$

$f_{1}(0, z)=\int_{\mathcal{P}} u_{x}(0, x, y, z) d x d y, f_{2}(0, z)=\int_{\mathcal{P}} u_{y}(0, x, y, z) d x d y$ and $f_{1}=$ $f_{2}=0$ at $z= \pm \frac{1}{2}$. For instance, if we assume that $R<R_{C}$ and that

$$
|\mathbf{g}(t)| \text { is small, } t \geq 0, \quad \text { and } \mathbf{g}(t) \rightarrow \mathbf{g}=\left(\begin{array}{l}
g_{1} \\
g_{2}
\end{array}\right), t \rightarrow+\infty, \quad \text { with } \mathbf{g} \neq 0,
$$


then all $\mathbf{u}$ in (I.1) converge to the Poiseuille flow

$$
-\frac{1}{2}\left(\begin{array}{c}
g_{1}\left(\frac{1}{4}-z^{2}\right) \\
g_{2}\left(\frac{1}{4}-z^{2}\right) \\
0
\end{array}\right) \text {. }
$$

This material is dealt with in Sec. III. One may believe that all these difficulties can be removed by requiring invariance properties of (I.1) against particular transformations of variables (Galileian invariance for instance). As we show in Sec. III this is not the case.

If we assume stress-free boundaries, the case of non-periodic pressure can be reduced to the periodic one by an appropriate change of variables. As a result we obtain for instance that under the assumptions (I.6) all velocity fields $\mathbf{u}$ in (I.1) approach an "accelerated" rigid motion.

There are several authors dealing with so called mean flows and nonperiodic pressure. We refer to $[\mathbf{C I}],[\mathbf{E D T S}],[\mathbf{I M}]$ and the references given there. In these papers the mean flow is given by $\int_{-1 / 2}^{+1 / 2} \int_{-\pi / \alpha}^{+\pi / \alpha} u_{y} d x d z$ for instance in the Taylor-Couette problem. In our considerations the situation is different. The mean flow of ours simply occurs as part of a particular decomposition of any solenoidal field $\mathbf{u}$ in the layer being periodic with respect to the $x, y$ directions and is given by $\mathbf{f}(z)=\int_{\mathcal{P}} \mathbf{u} d x d y$. Thus $\mathbf{f}$ enters in a very natural way into our access to the Boussinesq system. It satisfies a certain subsystem of (I.1) and exhibits a close connection with the pressure as was displayed in $[\mathbf{S v W I}]$. The details are given in the section to follow.

\section{Poloidal-Toroidal-Mean Flow Decomposition.}

We now briefly explain the poloidal-toroidal-mean flow decomposition of a solenoidal field in an infinite layer and the form (I.1) takes when using this decomposition. Any solenoidal field $\mathbf{u}$ in $\mathbb{R}^{2} \times\left(-\frac{1}{2}, \frac{1}{2}\right)$ which is $x, y$-periodic with respect to $\mathcal{P}$ can be decomposed into

$$
\mathbf{u}=\operatorname{curl} \operatorname{curl}(\varphi \mathbf{k})+\operatorname{curl}(\psi \mathbf{k})+\mathbf{f} .
$$

$\mathbf{k}$ is the unit vector in $z$-direction and is orthogonal with respect to the layer. $\varphi, \psi$ are functions having the same periodicity as $\mathbf{u}$. Their mean values over $\mathcal{P}$ vanish for all $z$. The vector field $\mathbf{f}$ depends on $z$ only. Its third component $f_{3}$ is constant and vanishes if $\mathbf{u}$ satisfies rigid or stress-free boundary conditions at the walls of the layer $z= \pm \frac{1}{2}$. We use the notations

$$
\boldsymbol{\delta} \varphi=\operatorname{curl} \operatorname{curl} \varphi \mathbf{k}=\left(\begin{array}{c}
\partial_{x z} \varphi \\
\partial_{y z} \varphi \\
\left(-\Delta_{2}\right) \varphi
\end{array}\right)
$$




$$
\begin{aligned}
& \text { with }\left(-\Delta_{2}\right)=-\partial_{x}^{2}-\partial_{y}^{2} \text { as plane Laplacian, } \\
\varepsilon \psi= & \operatorname{curl} \psi \mathbf{k}=\left(\begin{array}{c}
\partial_{y} \psi \\
-\partial_{x} \psi \\
0
\end{array}\right) \\
\boldsymbol{\delta} \cdot(\text { vector field })= & \partial_{x z}(\text { first component of vector field })+\ldots, \\
& \text { and similarly for } \boldsymbol{\varepsilon} \cdot(\text { vector field }) .
\end{aligned}
$$

Stress-free boundary conditions turn out to be equivalent to

$$
\varphi=\partial_{z}^{2} \varphi=\partial_{z} \psi=\partial_{z} f_{1}=\partial_{z} f_{2}=0 \quad \text { at } z= \pm \frac{1}{2}
$$

whereas rigid ones correspond to

$$
\varphi=\partial_{z} \varphi=\psi=f_{1}=f_{2}=0 \quad \text { at } z= \pm \frac{1}{2} .
$$

(I.1) is now rewritten in terms of the unknown vector

$$
\Phi=\left(\varphi, \psi, \vartheta, f_{1}, f_{2}\right)^{T}
$$

$\vartheta$ is the same quantity as in (I.1) and satisfies $\vartheta=0$ at $z= \pm \frac{1}{2}$ in any case. This new system we want to deal with arises by forming curlcurl and curl of the left hand side of the Navier-Stokes part of (I.1) and then taking its (Euclidean) scalar product with $\mathbf{k}$. Then we obtain

$$
\left\{\begin{aligned}
(-\Delta)\left(-\Delta_{2}\right) \partial_{t} \varphi+\Delta^{2}\left(-\Delta_{2}\right) \varphi-\sqrt{R}\left(-\Delta_{2}\right) \vartheta+\boldsymbol{\delta} \cdot(\mathbf{u} \cdot \nabla \mathbf{u}) & =0 \\
\left(-\Delta_{2}\right) \partial_{t} \psi+(-\Delta)\left(-\Delta_{2}\right) \psi-\varepsilon \cdot(\mathbf{u} \cdot \nabla \mathbf{u}) & =0 \\
\operatorname{Pr} \partial_{t} \vartheta-\Delta \vartheta-\sqrt{R}\left(-\Delta_{2}\right) \varphi+\operatorname{Pr} \mathbf{u} \cdot \nabla \vartheta & =0 \\
\partial_{t} f_{1}+\left(-\partial_{z}^{2}\right) f_{1}+\frac{1}{|\mathcal{P}|} \int_{\mathcal{P}} \tilde{\mathbf{u}} \cdot \nabla \tilde{u}_{x} d x d y & =-g_{1}(t) \\
\partial_{t} f_{2}+\left(-\partial_{z}^{2}\right) f_{2}+\frac{1}{|\mathcal{P}|} \int_{\mathcal{P}} \tilde{\mathbf{u}} \cdot \nabla \tilde{u}_{y} d x d y & =-g_{2}(t)
\end{aligned}\right.
$$

with $\mathbf{u}=\boldsymbol{\delta} \varphi+\varepsilon \psi+\mathbf{f}$ as before and $\tilde{\mathbf{u}}=\boldsymbol{\delta} \varphi+\varepsilon \psi$ as that part of $\mathbf{u}$ which has vanishing mean value over $\mathcal{P} . g_{1}(t), g_{2}(t)$ are given in (I.5). In the case of rigid or stress-free boundary conditions we introduce the Hilbert space

$$
\mathcal{H}=L_{M}^{2}(\Omega) \times L_{M}^{2}(\Omega) \times L^{2}(\Omega) \times L^{2}\left(\left(-\frac{1}{2}, \frac{1}{2}\right)\right) \times L^{2}\left(\left(-\frac{1}{2}, \frac{1}{2}\right)\right)
$$


where $L_{M}^{2}(\Omega)=\left\{f \in L^{2}(\Omega) \mid \int_{\mathcal{P}} f d x d y=0\right\}$ and where $L^{2}\left(\left(-\frac{1}{2}, \frac{1}{2}\right)\right)$ is endowed with the scalar product $|\mathcal{P}| \int_{-1 / 2}^{+1 / 2} f \bar{g} d z$. It is not too difficult to show that (II.1) takes the form

$$
\mathcal{B} \partial_{t} \Phi+\mathcal{A} \Phi-\sqrt{R} \mathcal{C} \Phi+\mathcal{M}(\Phi, \Phi)=\mathcal{G}
$$

$\mathcal{B}$ is a diagonal strictly positive definite selfadjoint operator, acting on the periodic vectors in $\mathcal{H}$. $\mathcal{A}$ does the same but is strictly positive definite selfadjoint only for rigid boundaries, whereas under stress-free boundary conditions $\mathcal{A}$ is only nonnegative selfadjoint, which is due to the mean-flow part. If $L^{2}\left(\left(-\frac{1}{2}, \frac{1}{2}\right)\right)$ is replaced by the subspace of mean-value-free functions then $\mathcal{A}$ becomes strictly positive definite. Evidently $\mathcal{A}$ is also diagonal, whereas the symmetric operator $\mathcal{C}$ has the matrix form

$$
\left(\begin{array}{ccccc}
0 & 0 & \left(-\Delta_{2}\right) & 0 & 0 \\
0 & 0 & 0 & 0 & 0 \\
\left(-\Delta_{2}\right) & 0 & 0 & 0 & 0 \\
0 & 0 & 0 & 0 & 0 \\
0 & 0 & 0 & 0 & 0
\end{array}\right) .
$$

$\mathcal{M}(\Phi, \Phi)$ is a bilinear nonlinearity, and $-\mathcal{G}$ contains the gradient of the nonperiodic part of $\pi$. In particular the periodic part of the pressure is eliminated and the system (II.1) is almost local. The only nonlocal parts are the mean values in the subsystem for the mean flow. For this material we refer to $[\mathbf{S v W I}]$. A certain technical difficulty occurs in the case of rigid boundary conditions. It is due to the fact that the operators $(-\Delta)$ in front of $\left(-\Delta_{2}\right) \partial_{t} \varphi$ and $\Delta^{2}$ in front of $\left(-\Delta_{2}\right) \varphi$ do not commute. To say it in other words: $\mathcal{B}$ and $\mathcal{A}$ do not commute. For this reason it is convenient to apply $(-\Delta)^{-1}$ under boundary conditions $\varphi=0$ at $z= \pm \frac{1}{2}$ to the first row in (II.1). Thus there arises

$$
\left(-\Delta_{2}\right) \partial_{t} \varphi+(-\Delta)^{-1} \Delta^{2}\left(-\Delta_{2}\right) \varphi-\sqrt{R}(-\Delta)^{-1}\left(-\Delta_{2}\right) \vartheta+(-\Delta)^{-1} \boldsymbol{\delta} \cdot(\mathbf{u} \cdot \nabla \mathbf{u})=0
$$

As the underlying Hilbert space for $\varphi$ we now take $\stackrel{\circ}{H}_{M}^{1,2}(\Omega)=\left\{\varphi \in H_{M}^{1,2}(\Omega) \mid\right.$ $\varphi=0$ at $\left.z= \pm \frac{1}{2}\right\}$. As $H_{M}^{1,2}(\Omega)$ we define in our situation the Hilbert space of all $f$,

$$
f(x, y, z)=\sum_{\kappa \in \mathbb{Z}^{2} \backslash\{0\}} a_{\kappa}(z) e^{i \alpha \kappa_{1} x+i \beta \kappa_{2} y}
$$

with

$$
\|f\|_{1}^{2}:=\|f\|_{H_{M}^{1,2}(\Omega)}^{2}
$$




$$
:=\sum_{\kappa \in \mathbb{Z}^{2} \backslash\{0\}}\left(\int_{-\frac{1}{2}}^{\frac{1}{2}}\left|\partial_{z} a_{\kappa}\right|^{2} d z+\left(\alpha^{2} \kappa_{1}^{2}+\beta^{2} \kappa_{2}^{2}\right) \int_{-\frac{1}{2}}^{\frac{1}{2}}\left|a_{\kappa}\right|^{2} d z\right) .
$$

Thus $\stackrel{\circ}{H}_{M}^{1,2}(\Omega)$ is endowed with the scalar-product $(\nabla \cdot \nabla \cdot)$. Then $\hat{A}=$ $(-\Delta)^{-1} \Delta^{2}$ is essentially selfadjoint in $\stackrel{\circ}{H}_{M}^{1,2}(\Omega)$ when defined on all $\varphi \in$ $H^{4,2}(\Omega) \cap \stackrel{\circ}{H}_{M}^{1,2}(\Omega)$ with $\partial_{z} \varphi=0$ at $z= \pm \frac{1}{2}$. Its closure $\overline{\hat{A}}$ is thus selfadjoint in $\stackrel{\circ}{H}_{M}^{1,2}(\Omega)$ with domain of definition $\left\{\varphi \in H^{3,2}(\Omega) \cap \stackrel{\circ}{H_{M}^{1,2}}(\Omega) \mid \partial_{z} \varphi=\right.$ 0 at $\left.z= \pm \frac{1}{2}\right\}$. The graph-norm of $\overline{\hat{A}}$ is equivalent with the $\|\cdot\|_{H^{3,2}(\Omega)}$-norm. $\overline{\hat{A}}$ is strictly positive definite in $\stackrel{\circ}{H}_{M}^{1,2}(\Omega)$, its square root $\hat{\hat{A}}^{1 / 2}$ has domain of definition $\left\{\varphi \in H^{2,2}(\Omega) \cap \stackrel{\circ}{H} \stackrel{1,2}{M}(\Omega) \mid \partial_{z} \varphi=0\right.$ at $\left.z= \pm \frac{1}{2}\right\}$. The norm $\left\|\hat{\hat{A}}^{1 / 2} \cdot\right\|$ is equivalent with the $\|\cdot\|_{H^{2,2}(\Omega)}$-norm. For this material we refer to [WI, Sec. V], [WII, Sec. V] and [KvWI, Sec. 0 and appendix]. The underlying Hilbert space under rigid boundary conditions now becomes

$$
\hat{\mathcal{H}}=\stackrel{\circ}{H}_{M}^{1,2}(\Omega) \times L_{M}^{2}(\Omega) \times L^{2}(\Omega) \times L^{2}\left(\left(-\frac{1}{2}, \frac{1}{2}\right)\right) \times L^{2}\left(\left(-\frac{1}{2}, \frac{1}{2}\right)\right)
$$

and (II.1) takes the form

$$
\hat{\mathcal{B}} \partial_{t} \Phi+\hat{\mathcal{A}} \Phi-\sqrt{R} \hat{\mathcal{C}} \Phi+\hat{\mathcal{M}}(\Phi, \Phi)=0
$$

Here $\hat{\mathcal{B}}, \hat{\mathcal{A}}$ commute and if $\mathcal{J}$ is the diagonal operator with $(-\Delta)^{-1}$ in the first position and the identity in all others then

$$
\hat{\mathcal{B}}=\mathcal{J} \mathcal{B}, \quad \hat{\mathcal{A}}=\mathcal{J} \mathcal{A}, \quad \hat{\mathcal{C}}=\mathcal{J C}, \quad \hat{\mathcal{M}}=\mathcal{J} \mathcal{M}
$$

$\hat{\mathcal{B}}, \hat{\mathcal{A}}$ are strictly positive definite selfadjoint operators in $\hat{\mathcal{H}}$. We set

$$
\hat{\mathcal{D}}=\left(\begin{array}{ccccc}
\overline{\hat{A}}^{1 / 2}\left(-\Delta_{2}\right) & 0 & 0 & 0 & 0 \\
0 & (-\Delta)^{1 / 2}\left(-\Delta_{2}\right) & 0 & 0 & 0 \\
0 & 0 & \sqrt{\operatorname{Pr}}(-\Delta)^{1 / 2} & 0 & 0 \\
0 & 0 & 0 & \left(-\partial_{z}^{2}\right)^{1 / 2} & 0 \\
0 & 0 & 0 & 0 & \left(-\partial_{z}^{2}\right)^{1 / 2}
\end{array}\right) .
$$

Then $\hat{\mathcal{D}}$ is in an obvious way an intermediate operator between $\hat{\mathcal{B}}$ and $\hat{\mathcal{A}}$; more precisely we can set $\hat{\mathcal{D}}=(\hat{\mathcal{B}}, \hat{\mathcal{A}})_{1 / 2}$ and in (II.3) we have

$$
\left(\hat{\mathcal{B}} \partial_{t} \Phi, \hat{\mathcal{A}} \Phi\right)=\frac{1}{2} \frac{d}{d t}\|\hat{\mathcal{D}} \Phi\|^{2}
$$




\section{Rigid Motions in the Periodic Pressure Case.}

We consider first the case of stress-free boundaries. The pressure $\pi$ is assumed to be periodic. By integrating (I.1) over $\Omega$ we obtain

$$
\frac{d}{d t} \frac{1}{|\Omega|} \int_{\Omega}\left(\begin{array}{l}
u_{1}(t, \mathbf{x}) \\
u_{2}(t, \mathbf{x})
\end{array}\right) d \mathbf{x}=-\frac{1}{|\Omega|} \int_{\Omega}\left(\begin{array}{l}
\partial_{x} \pi(t, \mathbf{x}) \\
\partial_{y} \pi(t, \mathbf{x})
\end{array}\right) d \mathbf{x}=0,
$$

or

$$
\frac{1}{|\Omega|} \int_{\Omega} u_{j}(t, \mathbf{x}) d \mathbf{x}=\frac{1}{|\Omega|} \int_{\Omega} u_{j}(0, \mathbf{x}) d \mathbf{x}=: c_{j} \in \mathbb{R}, \quad j=1,2 .
$$

Note that $\int_{\Omega} u_{3}(t, \mathbf{x}) d \mathbf{x}=0$ because of $\nabla \cdot \mathbf{u}=0$ and the boundary conditions. Instead of requiring $\left(c_{1}, c_{2}\right)^{\mathrm{T}}$ to vanish as in (I.2), we want to investigate the case $\left(c_{1}, c_{2}\right)^{T} \neq(0,0)^{\mathrm{T}}$, i.e. when rigid motions occur.

In order to stick to our usual Hilbert space $\mathcal{H}$ with zero-averaged mean flow components we consider $(\mathbf{u}, \pi, \vartheta)$ as perturbation of the constant flow $\mathbf{c}=\left(c_{1}, c_{2}, 0\right)^{\mathrm{T}}, \pi=0, \vartheta=0$, with disturbance $(\mathbf{w}, \pi, \vartheta), \mathbf{w}=\mathbf{u}-\mathbf{c}$. By expressing $(\mathbf{w}, \vartheta)$ in terms of $\Phi=\left(\varphi, \psi, \vartheta, f_{1}, f_{2}\right)^{\mathrm{T}}$ and writing $\Phi_{\mathbf{c}}=$ $\left(0,0,0, c_{1}, c_{2}\right)^{T}$ we have

$$
\left\{\begin{aligned}
\mathcal{B} \partial_{t} \Phi+\mathcal{A} \Phi-\sqrt{R} \mathcal{C} \Phi+\mathcal{M}\left(\Phi_{\mathbf{c}}, \Phi\right)+\mathcal{M}(\Phi, \Phi) & =0 \\
\Phi(0) & =\Phi_{0}
\end{aligned}\right.
$$

with stress-free boundary conditions for $\Phi$.

Due to the absence of $\mathcal{M}\left(\Phi, \Phi_{\mathbf{c}}\right)(=0)$ the energy $\left\|\mathcal{B}^{1 / 2} \Phi(t)\right\|^{2}$ behaves in exactly the same way as if $\Phi$ was a perturbation of the motionless state. In particular it decreases monotonically and exponentially provided $R<R_{C}$. The antisymmetric structure of $\mathcal{M}\left(\Phi_{\mathbf{c}}, \Phi\right)=\left(c_{1} \partial_{x}+c_{2} \partial_{y}\right) \mathcal{B} \Phi$ is responsible for the validity of the following lemma.

Lemma III.1. For every $\kappa \in \mathbb{Z}^{2} \backslash\{0\}$ satisfying

$$
\frac{\left(\pi^{2}+\alpha^{2} \kappa_{1}^{2}+\beta^{2} \kappa_{2}^{2}\right)^{3}}{\alpha^{2} \kappa_{1}^{2}+\beta^{2} \kappa_{2}^{2}}=R_{C}
$$

the eigenvector $\Phi=(\varphi, 0, \vartheta, 0,0)^{\mathrm{T}}$ of $\mathcal{A} \Phi-\sqrt{R_{C}} \mathcal{C} \Phi=0$ given by

$$
\left\{\begin{array}{l}
\varphi(x, y, z)=e^{i \alpha \kappa_{1} x+i \beta \kappa_{2} y} \cos \pi z \\
\vartheta(x, y, z)=\left(\left(\pi^{2}+\alpha^{2} \kappa_{1}^{2}+\beta^{2} \kappa_{2}^{2}\right)\left(\alpha^{2} \kappa_{1}^{2}+\beta^{2} \kappa_{2}^{2}\right)\right)^{1 / 2} \varphi(x, y, z)
\end{array}\right.
$$

simultaneously is an eigenvector of

$$
\sigma \mathcal{B} \Phi=\mathcal{A} \Phi-\sqrt{R_{C}} \mathcal{C} \Phi+\mathcal{M}\left(\Phi_{\mathbf{c}}, \Phi\right)
$$

with $\sigma=i\left(\alpha \kappa_{1} c_{1}+\beta \kappa_{2} c_{2}\right)$. 
Proof. Obvious.

Lemma III.1 together with [WIII, Thm. 1.1] implies that the marginal case $R=R_{C}$ for monotonic energy stability simultaneously is critical for linear stability analysis:

$$
\xi_{0}:=\inf \{\operatorname{Re} \sigma \mid \exists \text { eigenvector } \Phi \text { of (III.5) }\}=0,
$$

just as it was the case for the motionless state. With respect to disturbances $(\mathbf{w}, \vartheta)$ satisfying $\int_{\Omega} \mathbf{w}(t, \mathbf{x}) d \mathbf{x}=0$ the rigid motion $(\mathbf{c}, 0)$ therefore is unconditionally monotonically energy stable up to criticality.

Moreover the eigenspace $\left\{\Phi \mid \mathcal{A} \Phi-\sqrt{R_{C}} \mathcal{C} \Phi=0\right\}$ is spanned by the set of all vectors $\Phi$ given in (III.4), (III.3). From Lemma III.1 and [WIII, Lem. 1.1] we infer that

$$
\begin{aligned}
& \left\{\Phi \mid \mathcal{A} \Phi-\sqrt{R_{C}} \mathcal{C} \Phi=0\right\} \\
& =\operatorname{span}\left\{\Phi \mid \sigma \mathcal{B} \Phi=\mathcal{A} \Phi-\sqrt{R_{C}} \mathcal{C} \Phi+\mathcal{M}\left(\Phi_{\mathbf{c}}, \Phi\right)\right. \\
& \text { for some } \sigma \in \mathbb{C} \text { with } \operatorname{Re} \sigma=0\} \\
& =\bigoplus_{\substack{\eta=\alpha \kappa_{1} c_{1}+\beta \kappa_{2} c_{2} \\
\kappa \text { as in (III.3) }}}\left\{\Phi \mid i \eta \mathcal{B} \Phi=\mathcal{A} \Phi-\sqrt{R_{C}} \mathcal{C} \Phi+\mathcal{M}\left(\Phi_{\mathbf{c}}, \Phi\right)\right\} .
\end{aligned}
$$

In contrast to the motionless case, where $\sigma=0$ is the only eigenvalue of (III.5) with $\operatorname{Re} \sigma=0$ and therefore the principle of exchange of stability holds, this space of eigenvectors can now be split into several subspaces belonging to purely imaginary eigenvalues $i \eta$. To be more precise: $\sigma=0$ remains an eigenvalue of (III.5) if and only if the ratio $c_{1}: c_{2}$ and the ratio $-\beta \kappa_{2}: \alpha \kappa_{1}$ coincide for one of the $\kappa$ given by (III.3). Thus there are only finitely many possibilities for the ratio $c_{1}: c_{2}$ to produce the eigenvalue $\sigma=0$; in all other cases criticality is attained exclusively at purely imaginary eigenvalues. In particular this means that for almost all values of $c_{1}: c_{2}$ an exchange of stability no longer takes place.

The behaviour of $(\mathbf{u}, \vartheta)$ in relation to $(\mathbf{c}, 0)$ can as well be described by means of a Galileian transformation (cf. also (IV.7)):

$$
\tilde{\mathbf{u}}(t, \mathbf{x})=\mathbf{u}(t, \mathbf{x}+t \mathbf{c})-\mathbf{c}, \tilde{\vartheta}(t, \mathbf{x})=\vartheta(t, \mathbf{x}+t \mathbf{c}), \tilde{\pi}(t, \mathbf{x})=\pi(t, \mathbf{x}+t \mathbf{c})
$$

also solve (I.1) with stress-free boundaries, but with $\int_{\Omega} \tilde{\mathbf{u}}(t, \mathbf{x}) d \mathbf{x}=0$. Because of periodicity we have

$$
\|\tilde{\mathbf{u}}(t, \cdot)\|=\|\mathbf{u}(t, \cdot+t \mathbf{c})-\mathbf{c}\|=\|\mathbf{w}(t, \cdot)\|
$$


for every norm $\|\cdot\|$ involving plain integration over $\Omega$, hence the difference $(\mathbf{w}, \vartheta)=(\mathbf{u}, \vartheta)-(\mathbf{c}, 0)$ must behave in exactly the same way as the disturbance $(\tilde{\mathbf{u}}, \tilde{\vartheta})$ of the motionless state. For instance we get that monotonic energy stability of $(\mathbf{c}, 0)$ for $R \leq R_{C}$ is followed by nonlinear instability at $R>R_{C}$, since this is known to be true for the motionless case, cf. [ $\mathbf{Z}$, Satz 4], whereas the above method does not seem to provide this result without further effort.

The value of the first approach, however, consists in a precise description of the spectrum for the linearized problem at criticality, thereby presenting a simple example of the rare situation where criticality is realized by purely imaginary eigenvalues and linearized stability coincides with monotonic energy stability. The loss of exchange of stability becomes nevertheless more clear with the help of the Galileian transformation: The application of the inverse of (III.6) to $x, y$-dependent steady states (e.g. convection rolls) bifurcating from the rest state at $R=R_{C}$ leads to in general instationary solutions.

Finally we briefly address the case of (I.1) together with rigid boundary conditions. Here (III.1) does not hold, and there is no freedom of choice for $\int_{\Omega} \mathbf{u}(t, \mathbf{x}) d \mathbf{x}$, either, but it is determined uniquely by the mean-flow components of the rewritten Oberbeck-Boussinesq equations (II.2), due to the zero boundary values of $f_{1}, f_{2}$. In order to observe drift-like steady states in the case of rigid boundaries one must allow the pressure to be non-periodic. This will be dealt with in the following section.

\section{Non-Periodic Pressure.}

A non-periodic pressure $\pi$ with periodic gradient necessarily takes the form

$$
\pi(t, x, y, z)=\hat{\pi}(t, x, y, z)+g_{1}(t) x+g_{2}(t) y
$$

$\hat{\pi}$ being periodic, $g_{1}(t), g_{2}(t)$ independent of $x, y, z$, see (I.5). Here we want to show that in the case of rigid boundaries the behaviour of a solution $(\mathbf{u}, \pi, \vartheta)$ to (I.1) for $t \rightarrow \infty$ is governed by the behaviour of a solution $\mathbf{u}_{m}(t, z)$ to the parabolic mean field equation in one space variable

$$
\left\{\begin{array}{rlrl}
\partial_{t} u_{m, j}+\left(-\partial_{z}^{2}\right) u_{m, j} & =-g_{j}(t) & & \text { in }(0, \infty) \times\left(-\frac{1}{2}, \frac{1}{2}\right), \\
u_{m, j}\left(t, \pm \frac{1}{2}\right) & =0 & & \text { for } t>0,
\end{array} \quad j=1,2\right.
$$

provided that $R<R_{C}$ and that $\mathbf{u}_{m}$ stays small in a sense specified below. Note that $\left(\mathbf{u}_{m}, \vartheta_{m}\right)$ with $u_{m, 3}=0, \vartheta_{m}=0, \pi_{m}(t, x, y, z)=g_{1}(t) x+g_{2}(t) y$ solves (I.1), too. 
First we bound the energy in an appropriate way. Since the disturbance $\tilde{\mathbf{u}}=\mathbf{u}-\mathbf{u}_{m}, \tilde{\vartheta}=\vartheta-\vartheta_{m}=\vartheta$ also satisfies the rigid boundary conditions, we can write $(\tilde{\mathbf{u}}, \tilde{\vartheta})$ in terms of $\Phi=\left(\varphi, \psi, \vartheta, f_{1}, f_{2}\right)^{\mathrm{T}}$ as in $[\mathbf{S v W I}]$. The equation for $\Phi$ then reads

$$
\left\{\begin{aligned}
\mathcal{B} \partial_{t} \Phi+\mathcal{A} \Phi-\sqrt{R} \mathcal{C} \Phi+\mathcal{M}\left(\Phi, \Phi_{m}\right)+\mathcal{M}\left(\Phi_{m}, \Phi\right)+\mathcal{M}(\Phi, \Phi) & =0 \\
\Phi(0) & =\Phi_{0}
\end{aligned}\right.
$$

with $\Phi_{m}=\left(0,0,0, u_{m, 1}, u_{m, 2}\right)^{T}$, and the boundary conditions are, as usual,

$$
\varphi=\partial_{z} \varphi=0, \psi=0, \vartheta=0, f_{1}=f_{2}=0 \quad \text { at } z= \pm \frac{1}{2} .
$$

Observe that the pressure in the equation for $(\tilde{\mathbf{u}}, \tilde{\vartheta})$ is now periodic. By means of the energy method we obtain:

Theorem IV.1. Suppose that

$$
\sup _{\substack{0 \neq \Phi \in D\left(\mathcal{A}^{1 / 2}\right) \\ 0 \leq t<\infty}} \frac{\sqrt{R}(\mathcal{C} \Phi, \Phi)-\operatorname{Re}\left(\mathcal{M}\left(\Phi, \Phi_{m}\right), \Phi\right)}{\left\|\mathcal{A}^{1 / 2} \Phi\right\|^{2}}=: \rho<1 .
$$

Then there exists a constant $\gamma=\gamma(\operatorname{Pr}, \alpha, \beta, 1-\rho)>0$ such that for every strong solution $\Phi$ of (IV.3) the energy $\left\|\mathcal{B}^{1 / 2} \Phi(t)\right\|^{2}$ is bounded by

$$
\left\|\mathcal{B}^{1 / 2} \Phi(t)\right\|^{2} \leq\left\|\mathcal{B}^{1 / 2} \Phi(s)\right\|^{2} \leq e^{-\gamma s}\left\|\mathcal{B}^{1 / 2} \Phi_{0}\right\|^{2}, \quad 0 \leq s \leq t<T\left(\Phi_{0}\right),
$$

in its maximal interval of existence $\left(0, T\left(\Phi_{0}\right)\right)$. A sufficient condition for (IV.4) to hold is

$$
\sqrt{\frac{R}{R_{C}}}+\frac{\sup _{0 \leq t<\infty,|z| \leq \frac{1}{2}}\left|\partial_{z} \mathbf{u}_{m}(t, z)\right|}{\sqrt{2}\left(\pi^{2}+\min \left\{\alpha^{2}, \beta^{2}\right\}\right)}<1
$$

Proof. (IV.5) follows as usual by multiplying (IV.3) by $\Phi$, taking the real part and integrating. In order to prove the sufficiency of (IV.6) we recall the estimate $|(\mathcal{C} \Phi, \Phi)| \leq{\sqrt{R_{C}}}^{-1}\left\|\mathcal{A}^{1 / 2} \Phi\right\|^{2}$, which is due to the definition of $R_{C}$ (cf. e.g. [SvWII, Sec. 2]). Thus we still must estimate $\left|\left(\mathcal{M}\left(\Phi, \Phi_{m}\right), \Phi\right)\right|$. Setting $\|f\|_{\mathcal{P}}^{2}:=\int_{\mathcal{P}}|f(x, y, z)|^{2} d x d y$ we have

$$
\left|\left(\mathcal{M}\left(\Phi, \Phi_{m}\right), \Phi\right)\right|
$$




$$
\begin{aligned}
= & \mid\left(\partial_{z} u_{m, 1}\left(-\Delta_{2}\right) \varphi, \partial_{x} \partial_{z} \varphi\right)+\left(\partial_{z} u_{m, 2}\left(-\Delta_{2}\right) \varphi, \partial_{y} \partial_{z} \varphi\right) \\
& +\left(\partial_{z} u_{m, 1}\left(-\Delta_{2}\right) \varphi, \partial_{y} \psi\right)+\left(\partial_{z} u_{m, 2}\left(-\Delta_{2}\right) \varphi,-\partial_{x} \psi\right) \mid \\
\leq & \int_{-\frac{1}{2}}^{\frac{1}{2}}\left(\frac{\left|\partial_{z} u_{m, 1}(t, z)\right|}{2}\left(\frac{2}{\varepsilon}\left\|\left(-\Delta_{2}\right) \varphi\right\|_{\mathcal{P}}^{2}+\varepsilon\left\|\partial_{x} \partial_{z} \varphi\right\|_{\mathcal{P}}^{2}+\varepsilon\left\|\partial_{y} \psi\right\|_{\mathcal{P}}^{2}\right)\right. \\
& \left.+\frac{\left|\partial_{z} u_{m, 2}(t, z)\right|}{2}\left(\frac{2}{\delta}\left\|\left(-\Delta_{2}\right) \varphi\right\|_{\mathcal{P}}^{2}+\delta\left\|\partial_{y} \partial_{z} \varphi\right\|_{\mathcal{P}}^{2}+\delta\left\|-\partial_{x} \psi\right\|_{\mathcal{P}}^{2}\right)\right) d z
\end{aligned}
$$

For $\varepsilon=\sqrt{2\left(1+\frac{\left|\partial_{z} u_{m, 2}(t, z)\right|^{2}}{\left|\partial_{z} u_{m, 1}(t, z)\right|^{2}}\right)}, \delta=\sqrt{2\left(1+\frac{\left|\partial_{z} u_{m, 1}(t, z)\right|^{2}}{\left|\partial_{z} u_{m, 2}(t, z)\right|^{2}}\right)}$ the right hand side equals

$$
\begin{aligned}
& \int_{-\frac{1}{2}}^{\frac{1}{2}} \frac{\left|\partial_{z} \mathbf{u}_{m}(t, z)\right|}{\sqrt{2}}\left(\|\boldsymbol{\delta} \varphi\|_{\mathcal{P}}^{2}+\|\varepsilon \psi\|_{\mathcal{P}}^{2}\right) d z \\
& \leq \sup _{|z| \leq \frac{1}{2}} \frac{\left|\partial_{z} \mathbf{u}_{m}(t, z)\right|}{\sqrt{2}}\left\|\mathcal{B}^{1 / 2}(\varphi, \psi, 0,0,0)^{\mathrm{T}}\right\|^{2} .
\end{aligned}
$$

The best constant $\mu$ in $\left\|\mathcal{B}^{1 / 2} \Phi\right\|^{2} \leq \mu\left\|\mathcal{A}^{1 / 2} \Phi\right\|^{2}, \Phi=(\varphi, \psi, 0,0,0)^{T} \in D\left(\mathcal{A}^{1 / 2}\right)$ turns out to be $\mu=\left(\pi^{2}+\min \left\{\alpha^{2}, \beta^{2}\right\}\right)^{-1}$, and the assertion is proved.

(IV.5) says that the instationary solution $\left(\mathbf{u}_{m}, \vartheta_{m}\right)$ of (I.1) is monotonically and unconditionally energy stable with exponentially decaying disturbances, provided that (IV.4) holds. The quantity $\sup _{t, z}\left|\partial_{z} \mathbf{u}_{m}(t, z)\right|$ in (IV.6) can be considered as Reynolds number. For $g_{1}(t) \equiv g_{1}, g_{2}(t) \equiv 0$ and $\Phi_{m}=\left(0,0,0,-\frac{1}{2} g_{1}\left(\frac{1}{4}-z^{2}\right), 0\right)^{T}$ being the corresponding Poiseuille flow (IV.4) is just a reformulation of [Mu, (3.14)].

Concerning higher norms we can further show:

Lemma IV.2. Assume that (IV.4) holds and that the norms $\left\|\partial_{t} \mathbf{u}_{m}(t, \cdot)\right\|_{L^{2}\left(\left(-\frac{1}{2}, \frac{1}{2}\right)\right)}$ and $\left\|\partial_{z}^{2} \mathbf{u}_{m}(t, \cdot)\right\|_{L^{2}\left(\left(-\frac{1}{2}, \frac{1}{2}\right)\right)}$ are bounded uniformly in time t. If $\left\|\hat{\mathcal{D}} \Phi_{0}\right\|_{\hat{\mathcal{H}}}$ is sufficiently small then the strong solution $\Phi$ exists globally, i.e. $T\left(\Phi_{0}\right)=+\infty, \Phi_{m}$ is locally conditionally stable with respect to the disturbance $\Phi$ in the norm $\|\hat{\mathcal{A}} \cdot\|_{\hat{\mathcal{H}}}$, and $\|\hat{\mathcal{A}} \Phi(t)\|_{\hat{\mathcal{H}}} \rightarrow 0$ for $t \rightarrow \infty$.

Proof. The proofs of [KvWI, Thm. 1.1, Thm. 2.1, Thm. 3.1] and [KvWII, Thm. 2.1 and remark thereafter] carry over to the case of time-dependent $\Phi_{m}$ if the required bounds for $\Phi_{m}$ are uniform in time. This, however, is guaranteed by our assumption.

The asymptotic behaviour of $(\mathbf{u}, \vartheta)$ is therefore determined by the behaviour of the mean flow solution $\mathbf{u}_{m}$ even in higher norms. 
Suppose, for instance, that $\mathbf{g}(t)=\left(g_{1}(t), g_{2}(t), 0\right)^{T}$ is continuous in $t$ with $\mathbf{g}(t) \rightarrow \mathbf{g}=\left(g_{1}, g_{2}, 0\right)^{T}$ as $t \rightarrow \infty$, and that $R<R_{C}$. Then every solution of the mean field system (IV.2) converges to the stationary field

$$
\mathbf{u}_{p}(z)=-\frac{1}{2}\left(\frac{1}{4}-z^{2}\right)\left(\begin{array}{c}
g_{1} \\
g_{2} \\
0
\end{array}\right)
$$

which is a Poiseuille flow. If we prescribe as initial values e.g. $u_{m, j}(0, z)=$ $-\frac{1}{2} g_{j}(0)\left(\frac{1}{4}-z^{2}\right)$ we see that $\left|\partial_{z}^{2} \mathbf{u}_{m}(t, z)\right| \leq \sqrt{2} \sup _{t}|\mathbf{g}(t)|$, thus $\left|\partial_{z} \mathbf{u}_{m}(t, z)\right| \leq$ $\sqrt{2} \sup _{t}|\mathbf{g}(t)|$ for every $(t, z)$. Hence if $|\mathbf{g}(t)|$ remains small, cf. (IV.6), the total flow $(\mathbf{u}, \vartheta)$ also converges to the Poiseuille flow $\left(\mathbf{u}_{p}, \vartheta_{p}=0\right)$ with respect to the energy norm, irrespective of the size of the initial value $(\mathbf{u}(0, \cdot), \vartheta(0, \cdot))$. From Lemma IV.2 we conclude that $\left(\mathbf{u}_{p}, 0\right)$ is conditionally asymptotically stable with respect to the higher norm $\|\hat{\mathcal{A}} \cdot\|_{\hat{\mathcal{H}}}$, too. The smallness of $\left\|\hat{\mathcal{D}} \Phi_{0}\right\|_{\hat{\mathcal{H}}}$ required there guarantees the global existence of $(\mathbf{u}, \vartheta)$ as strong solution. By considering $(\mathbf{u}, \vartheta)$ as weak solution to (IV.1), however, one still can show that $(\mathbf{u}, \vartheta)$ must converge to the Poiseuille flow $\left(\mathbf{u}_{p}, 0\right)$ in $\|\hat{\mathcal{A}} \cdot\|_{\hat{\mathcal{H}}}$, even if the smallness assumption for $\left\|\hat{\mathcal{D}} \Phi_{0}\right\|_{\hat{\mathcal{H}}}$ is dropped, see [KvWII, Sec. 3] for a detailed discussion.

The case of non-periodic pressure and stress-free boundaries could be treated in a similar way to the one used above, here one would have to replace the Dirichlet problem in (IV.2) by the corresponding Neumann problem. It is however possible to describe the situation for stress-free boundaries even more exactly by making use of a transformation leaving (I.5) invariant, cf. [M, p. 15]: If $(\mathbf{u}, \pi, \vartheta)$ solves (I.1) with stress-free boundaries and if $\boldsymbol{\xi}(t)=\left(\xi_{1}(t), \xi_{2}(t), 0\right)^{T}$ and $q(t)$ are smooth then the functions

$$
\left\{\begin{array}{l}
\tilde{\mathbf{u}}(t, \mathbf{x})=\mathbf{u}(t, \mathbf{x}-\boldsymbol{\xi}(t))+\boldsymbol{\xi}^{\prime}(t), \\
\tilde{\vartheta}(t, \mathbf{x})=\vartheta(t, \mathbf{x}-\boldsymbol{\xi}(t)), \\
\tilde{\pi}(t, \mathbf{x})=\pi(t, \mathbf{x}-\boldsymbol{\xi}(t))+q(t)-\xi_{1}^{\prime \prime}(t) x-\xi_{2}^{\prime \prime}(t) y
\end{array}\right.
$$

solve (I.1) with stress-free boundaries as well. Observe that this is not true for the case of rigid boundaries because of $\boldsymbol{\xi}^{\prime}(t) \neq 0$ at $z= \pm \frac{1}{2}$ unless $\boldsymbol{\xi}(t)=$ const. In the stress-free case we now choose

$$
\boldsymbol{\xi}(t)=-\frac{t}{|\Omega|} \int_{\Omega} \mathbf{u}(0, \mathbf{x}) d \mathbf{x}+\int_{0}^{t} \int_{0}^{\tau}\left(\begin{array}{c}
g_{1}(s) \\
g_{2}(s) \\
0
\end{array}\right) d s d \tau
$$

and $q(t)=g_{1}(t) \xi_{1}(t)+g_{2}(t) \xi_{2}(t), g_{1}(t), g_{2}(t)$ being determined from $\pi$ by (IV.1). Then $(\tilde{\mathbf{u}}, \tilde{\pi}, \tilde{\vartheta})$ solves (I.1) with $\tilde{\pi}$ being periodic and $\int_{\Omega} \tilde{\mathbf{u}}(t, \mathbf{x}) d \mathbf{x}=0$. 
The rigid motion $(\mathbf{v}, 0)$ given by

$$
\mathbf{v}(t, x, y, z)=\mathbf{v}(t)=\frac{1}{|\Omega|} \int_{\Omega} \mathbf{u}(0, \mathbf{x}) d \mathbf{x}-\int_{0}^{t}\left(\begin{array}{c}
g_{1}(\tau) \\
g_{2}(\tau) \\
0
\end{array}\right) d \tau=-\boldsymbol{\xi}^{\prime}(t)
$$

is transformed into $(\tilde{\mathbf{v}}, 0)=(0,0)$, i.e. the motionless state. For every norm $\|\cdot\|$ involving plain integration over $\Omega$ we have

$$
\|\mathbf{u}(t, \cdot)-\mathbf{v}(t)\|=\|\mathbf{u}(t, \cdot-\boldsymbol{\xi}(t))-\mathbf{v}(t)\|=\|\tilde{\mathbf{u}}(t, \cdot)\|
$$

due to periodicity. Hence the difference $(\mathbf{u}(t, \cdot), \vartheta(t, \cdot))-(\mathbf{v}(t), 0)$ exactly behaves like the disturbance $(\tilde{\mathbf{u}}, \tilde{\vartheta})$ of the rest state in (I.1). But this behaviour is well-investigated, cf. e.g. [SvWI], [SvWII], [KvWI], [KvWII], [WI], [WII], [WIII]. If, for instance, $R<R_{C}$ then $(\mathbf{u}, \vartheta)$ approaches the rigid motion $(\mathbf{v}(t), 0)$ unconditionally with respect to the energy norm. In particular the results from the end of Sec. II can be recovered by means of the above transformation.

\section{Conclusion.}

It has been studied for both rigid and stress-free boundaries, how the presence of non-periodic pressure affects the long time behaviour of solutions to the Oberbeck-Boussinesq equations. It turns out that the flow is governed entirely by the parabolic subsystem in one space variable for its mean flow components, provided that $R<R_{C}$ and the Reynolds number for the mean flow is not too large, cf. (IV.6). Rigid motion can also occur in the case of periodic pressure if the boundary conditions are stress-free. Linear stability analysis then shows that the energetic and the critical Rayleigh numbers still coincide, although the principle of exchange of stability fails to hold because the marginal case is realized by purely imaginary eigenvalues. This is a general effect which also appears if one considers disturbances to a constant horizontal flow and imposes rigid boundary conditions for the disturbance and periodicity for the corresponding pressure.

\section{References}

[CI] P. Chossat and G. Iooss, The Taylor-Couette problem, Springer, New York etc., 1994.

[ETDS] W.S. Edwards, R.P. Tagg, B.C. Dornblaser and H.L. Swinney, Periodic traveling waves with nonperiodic pressure, European J. Mech. B/Fluids, 10-Suppl. (1991), 205-210. 
[GP] G.P. Galdi and M. Padula, A new approach to the energy theory in the stability of fluid motion, Arch. Rational Mech. Anal., 110 (1990), 187-286.

[GS] G.P. Galdi and B. Straughan, Exchange of stabilities, symmetry, and nonlinear stability, Arch. Rational Mech. Anal., 89 (1985), 211-228.

[IM] G. Iooss and A. Mielke, Bifurcating time-periodic solutions of Navier-Stokes equations in infinite cylinders, J. Nonlinear Sci., 1 (1991), 107-146.

[KvWI] Y. Kagei and W. von Wahl, Stability of higher norms in terms of energy stability for the Boussinesq-equations. Remarks on the asymptotic behaviour of convectionroll-type solutions, Differential Integral Equations, 7 (1994), 921-948.

[KvWII _ _ Asymptotic stability of higher order norms in terms of asymptotic energy stability for viscous incompressible fluid flows heated from below, Japan J. Indust. Appl. Math., 13 (1996), 33-49.

[M] A. Mielke, Mathematical analysis of sideband instabilities with application to Rayleigh-Bénard convection, Preprint, University of Hannover, 1995.

[Mu] G. Mulone, On the stability of plane parallel convective flow, Acta Mech., 87 (1991), 153-162.

[SvWI] B.J. Schmitt and W. von Wahl, Decomposition of solenoidal fields into poloidal fields, toroidal fields and the mean flow. Applications to the Boussinesq-equations, The Navier-Stokes Equations II - Theory and Numerical Methods. Proceedings, Oberwolfach 1991, Lecture Notes in Math., 1530 (eds. J.G. Heywood, K. Masuda, R. Rautmann and S.A. Solonnikov), 291-305. Springer, Berlin, Heidelberg, New York, 1992.

[SvWII _ Monotonicity and boundedness in the Boussinesq-equations, European J. Mech. B/Fluids, 12 (1993), 245-270.

[WI] W. von Wahl, The Boussinesq-equations in terms of poloidal and toroidal fields and the mean flow, Lecture Notes, Bayreuth. Math. Schr., 40 (1992), 203-290.

[WII] - The Boussinesq-equations in terms of poloidal and toroidal fields and the mean flow, Lecture Notes, Improved and corrected version. Preprint, University of Bayreuth, 1992.

[WIII] Necessary and sufficient conditions for the stability of flows of incompressible viscous fluids, Arch. Rational Mech. Anal., 126 (1994), 103-129.

[Z] F. Zimmermann, Unbedingte Stabilität bis zur Kritikalität der axisymmetrischen Taylor-Couette-Strömung im Grenzfall kleiner Spaltbreite, Diploma thesis, University of Bayreuth, 1996.

Received January 27, 1997 and revised August 5, 1997.

RWTH AACHEN

LEHRSTUHL I FÜR MATHEMATIK

D-52056 Aachen, Germany

E-mail address: schmitt@math1.rwth-aachen.de

Department of Mathematics

UNIVERSITY OF BAYREUTH

D-95440 Bayreuth, Germany

E-mail address: wolf.vonwahl@uni-bayreuth.de 